\title{
Silver and Copper Microstructures: The Workfunction's Effect
}

\author{
Tahir Mehmood, Aiman Mukhtar* \\ The State Key Laboratory of Refractories and Metallurgy, Hubei Province Key Laboratory of Systems Science in Metallurgical Process, \\ International Research Institute for Steel Technology, Wuhan University of Science and Technology, Wuhan, P. R. China
}

Email address:

tahir10621@yahoo.com (T. Mehmood), aiman10603@yahoo.com (A. Mukhtar)

${ }^{*}$ Corresponding author

\section{To cite this article:}

Tahir Mehmood, Aiman Mukhtar. Silver and Copper Microstructures: The Workfunction's Effect. Modern Chemistry. Vol. 5, No. 1, 2017, pp. 1-6. doi: 10.11648/j.mc.20170501.11

Received: December 30, 2016; Accepted: January 24, 2017; Published: February 10, 2017

\begin{abstract}
To understand the growth mechanism of $\mathrm{Ag}$ and $\mathrm{Cu}$ nanowires we have studied the effect of workfunction on the metal nanowire growth by XRD, SEM and Potentiostat. Under the same potential and overpotential, the metal with a smaller workfunction has a higher current density, i.e. current density for $\mathrm{Ag}$ is higher than of $\mathrm{Cu}$ nanowires. Likewise metals, the plane with smaller workfunction grows faster than with the larger workfunction, thus the preferential growth plane is (220) for both metals. We argued that the current arises from electrons tunneling from metal surface to hydrated metal and hydrogen ions. The metal with a smaller workfunction has a thinner barrier for tunneling, thus leading to a higher current density. It is found that deposition method have no such effect on the structure of deposited nanowires.
\end{abstract}

Keywords: Workfunction, Electron Tunneling, Metal Nanowires, Growth

\section{Introduction}

Metal nanowires have attracted a great deal of research interest in recent years because of their electronic and optical properties $[1,2]$ and potential applications in nanodevices [3]. Several groups prepared and studied the silver nanowires or nanorods using templates [1-6]. Currently, the synthesis of nanowires by the electrochemical deposition is of great interest. To fabricate and investigate the growth mechanisms of metal nanowires in the cylindrical pores of a template is of essential and technological interests [7-12]. The template-assisted synthesis of nanowires is a conceptually simple and intuitive way to fabricate nanostructures $[13,14]$. Template commonly used for nanowire synthesis is Anodic Aluminum Oxide (AAO) owing to its salient features such as decorative properties, nanopore symmetry and corrosion resistance $[15,16]$. Understanding various factors that influence the growth of electrodeposited metal nanowires is of scientific and technological interests. Deposition potential plays an important role in controlling the metal nanowire growth. For example, the nanowires of $\mathrm{Au}, \mathrm{Ag}$, and $\mathrm{Cu}$ are single crystalline under the low deposition potential, and polycrystalline under the high deposition potential [17].

We have proposed in the previous paper that the growth of metal nanowires can be pictured as the four steps at the atomic-level scale [18]. The dehydration of hydrated metal ions can be the most important step among the four steps. The dehydration involves valence electrons tunneling to hydrated metal ions $[18,19]$, leading to neutralization of the hydrated metal ions. The neutral metal atoms are adsorbed on the surface and then diffuse to surface sites (such as kink site) where they incorporate into the metal lattice, thus leading to the growth [18]. Therefore, the growth rate is related by the dehydration [18]. Since the probability of electron tunneling is related to the workfunction of metals, the workfunction can have a significant effect on the growth of metal nanowires. Moreover, the elemental processes of electrodeposition are still not well understood. Therefore the present paper attempts to study the workfunction effect of metal nanowire growth and to understand the electrode reaction mechanism and also its effect the growth of planes of same metal. 


\section{Material and Methods}

The detailed experimental procedure for preparation of the porous anodic alumina oxide (AAO) templates has been given in our previous paper [18, 20-22]. The templates obtained by this procedure have the cylindrical and hexagonally arranged pores of about $50 \mathrm{~nm}$ in diameter. A gold $(\mathrm{Au})$ film was sputtered onto the back side of the templates to serve as the working electrode. The two electrolytes have the same concentration of metal ions. The electrolyte was $0.2 \mathrm{M} \mathrm{AgNO}_{3}$ and $0.68 \mathrm{M} \mathrm{H} \mathrm{H}_{3} \mathrm{BO}_{3}$ for deposition of $\mathrm{Ag}$ nanowires, and $0.2 \mathrm{M} \mathrm{CuSO}_{4}$ and $0.68 \mathrm{M}$ $\mathrm{H}_{3} \mathrm{BO}_{3}$ for deposition of $\mathrm{Cu}$ nanowires. The $\mathrm{pH}$ of electrolytes was adjusted to 2.5 by adding $1 \mathrm{M} \mathrm{H}_{2} \mathrm{SO}_{4}$ solution. Direct current electrodeposition was conducted in a three-electrode cell at room temperature. The area of the working electrode for growth of nanowires was $0.608 \mathrm{~cm}^{2}$ $\left(=0.25 \pi(0.88 \mathrm{~cm})^{2}\right)$ and the area of the graphite counter electrode was $14.7 \mathrm{~cm}^{2}(=4.2 \mathrm{~cm} \times 3.5 \mathrm{~cm})$. The reference electrode was the saturated calomel. Two different kinds of deposition were performed to understand the growth mechanism of $\mathrm{Ag}$ and $\mathrm{Cu}$ nanowires. First, the deposition was performed with a slow scan rate of $0.1 \mathrm{mV} / \mathrm{s}$. The potential ranges for $\mathrm{CV}$ were, 0.098 to $-1.2 \mathrm{~V}$ (SCE) for $\mathrm{Cu}$ and 0.558 to $-0.3 \mathrm{~V}$ for $\mathrm{Ag}$. The starting point of scanning is near the equilibrium potential of deposited metal. The other deposition was conducted at fixed potential of $-0.4 \mathrm{~V}$. The $\mathrm{Ag}$ and $\mathrm{Cu}$ nanowires were analyzed by $\mathrm{X}$-ray diffraction $(\mathrm{XRD}$, X'Pert PRO MRD, PANalytical, Netherlands) with $\mathrm{CuK}_{\alpha}$ radiation. Before XRD measurements the sputtered Au film and the over-deposition were mechanically polished away. The deposited $\mathrm{Cu}, \mathrm{Co}$ and $\mathrm{Ni}$ nanowires were also examined by scanning electron microscope (SEM, JEOL JSM-6700F). In order to perform SEM observations, the AAO template was partly dissolved with a $5 \mathrm{wt} \% \mathrm{NaOH}$ solution, and then carefully rinsed with deionized water for several times.

\section{Results}

Figure 1 demonstrates the current density vs time curves ( $I-t$ curves) for the deposition of $\mathrm{Ag}$ and $\mathrm{Cu}$ nanowires at fixed potential of $-0.4 \mathrm{~V}$. From the figure again one can see that at the same potential and at same experimental conditions the deposition current density for $\mathrm{Ag}$ is higher than that of $\mathrm{Cu}$. In the next section we will explain why the current density is higher for $\mathrm{Ag}$ nanowires and lower for $\mathrm{Cu}$ nanowires, also why $\mathrm{Ag}$ nanowires grow faster than of $\mathrm{Cu}$. In $\mathrm{CV}$ deposition there was a thick layer of $\mathrm{Ag}$ on the template that shows the faster growth of Ag nanowires, which was mechanically removed after the deposition completed. Also the growth rate can be realized from the $I-t$ curves, the time required for the deposition of $\mathrm{Ag}$ is very less than that of the $\mathrm{Cu}$ nanowires (see Figure 1).

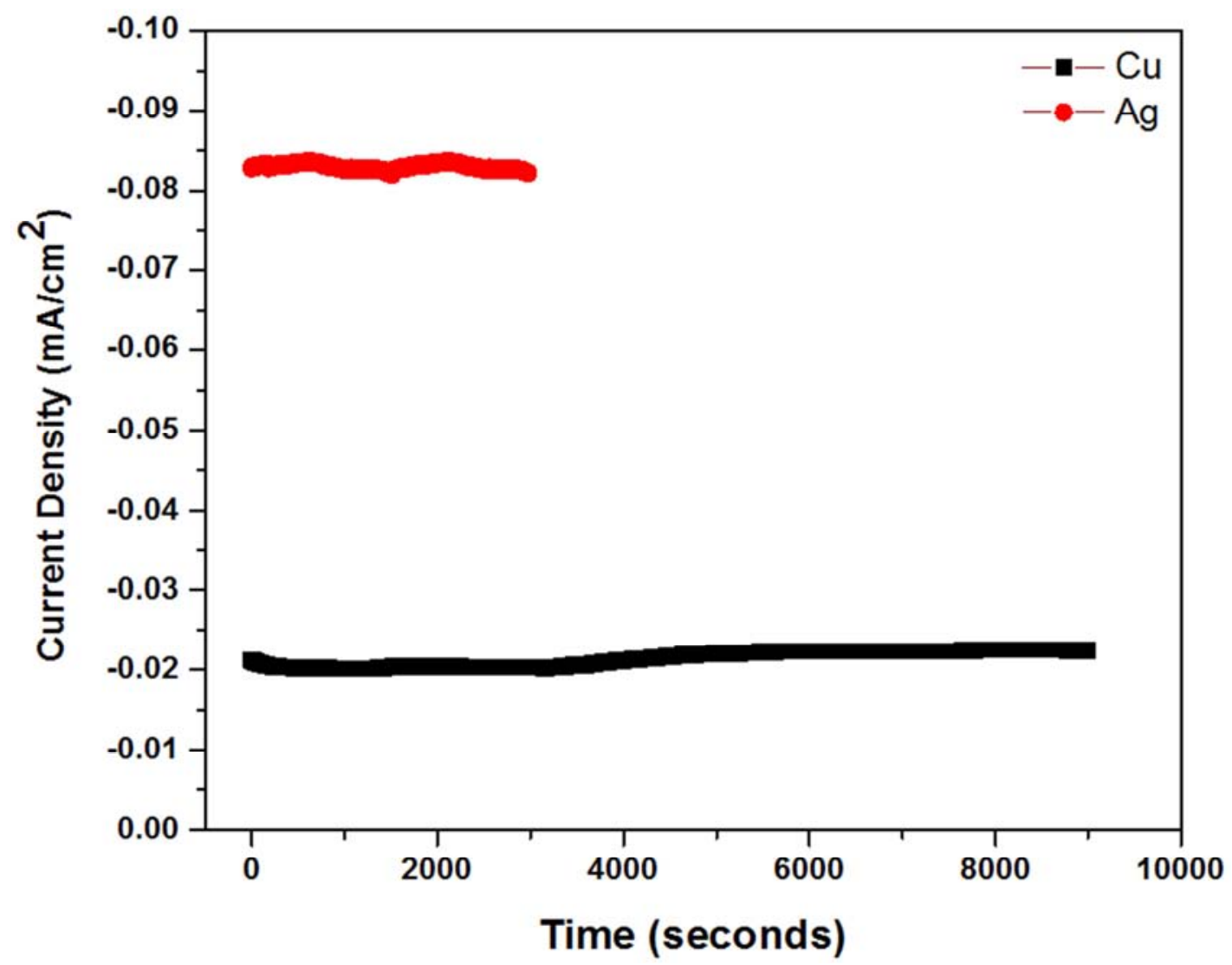

Figure 1. Current density vs time curves for Ag and Cu measured at fixed deposition potential -0.4V.

Figure 2 shows the XRD patterns of $\mathrm{Ag}$ and $\mathrm{Cu}$ nanowires deposited by two different deposition techniques (CV deposition, and deposition at fixed potential). The XRD patterns were collected from the top side of nanowire. The 
$\mathrm{Ag}$ and $\mathrm{Cu}$ nanowires were deposited at room temperature with scan rate of $0.1 \mathrm{mV} / \mathrm{s}$ for $\mathrm{CV}$ deposition, and at fixed potential of $-0.4 \mathrm{~V}$. From the figure it can be seen that there is no such difference in the structure of deposited nanowires for both $\mathrm{CV}$ and fixed potential deposition. The diffraction peaks are corresponding to (111) and (220) reflections of the facecentered cubic (fcc) structure of bulk Ag with the standard lattice constant of $\mathrm{a}=4.077 \AA$ (JCPDS $87-0720$ ), respectively. It is obvious that the intensity of peak at $64.36^{\circ}$ is much stronger than that at $38.18^{\circ}$ (111), which means that our silver nanowires have preferred (220) orientation. For $\mathrm{Cu}$, all peaks match well with the fcc $\mathrm{Cu}$ (JPCDS, 70-3038) with lattice constant $\mathrm{a}=3.625 \AA$. It can be seen that the preferential growth plane is fcc (220). For the same metal, different planes have different workfunction and the atomically rough surface has a smaller workfunction than the atomically smooth surface such as fcc (111).

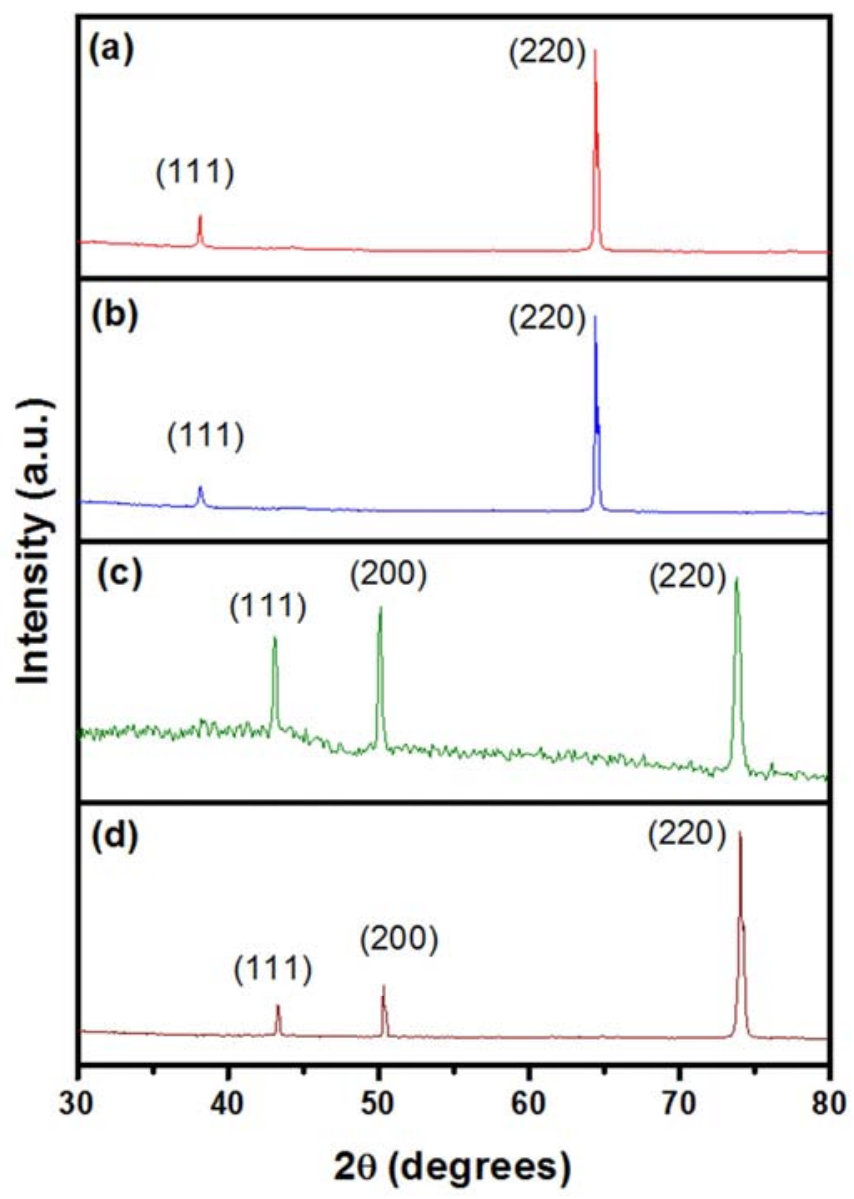

Figure 2. XRD patterns for electrodeposited nanowires, a) $\mathrm{Ag} C V$ deposition, b) $\mathrm{Ag}$ deposited at fixed potential $-0.4 \mathrm{~V}, \mathrm{c}$ ) $\mathrm{Cu} \mathrm{CV}$ deposition, b) $\mathrm{Cu}$ deposited at fixed potential $-0.4 \mathrm{~V}$.

For example, the workfunction of the (110) and (111) surfaces is $4.14(+0.04$ or -0.04$) \mathrm{eV}$ and $4.46(+0.02$ or -0.02$)$ $\mathrm{eV}$ for $\mathrm{Ag}$ [23], $4.48 \mathrm{eV}$ and $4.94 \mathrm{eV}$ for $\mathrm{Cu}$, respectively [24]. We can again see that the plane with a smaller workfunction grows faster (preferentially).

Figure 3 shows SEM images of nanowires deposited at fixed potential of $-0.4 \mathrm{~V}$ and a scan rate of $0.1 \mathrm{mV} / \mathrm{s}$ for $\mathrm{CV}$ deposition.

Figure 3 (a, and b) presents the Ag nanowires, Figure 3 (c, and $\mathrm{d}$ ) the $\mathrm{Cu}$ nanowires. The diameter of the deposited $\mathrm{Ag}$, and $\mathrm{Cu}$ nanowires $(\sim 50 \mathrm{~nm})$ is the same as that of the pores of AAO template $(\sim 50 \mathrm{~nm})$, indicating that the cylindrical pores of the AAO template were fully filled with $\mathrm{Ag}(\mathrm{Cu})$ atoms during the deposition.

\section{Discussion}

As described in the introduction section that growth of the nanowires is related to dehydration and the tunneling is related with the workfunction of metals. Here we discuss that how the workfunction significantly affect the growth of metal nanpwires within the pores of AAO template.

In electrodeposition of metal, a metal ion $\mathrm{M}^{n+}$ is transferred from solution into the ionic metal lattice, meanwhile electrons are provided from the external electron source (power supply) to the electron gas of the metal M [25]. When an adsorbed hydrated metal ion captures electrons from the surface by quantum-mechanical tunneling, the metal ion becomes a neutral metal atom. The electrostatic attractive interaction between the neutral metal atom and water molecules is zero and the water molecules of hydration are displaced. The neutral metal atom adsorbed on the surface and diffuses to a surface site (such as kink site) where it incorporates into the ionic metal lattice, leading to the growth of nanowires. The current density arises from valence electrons tunneling from metal surface tunneling to hydrated metal and hydrogen ions, leading metal nanowire growth and hydrogen evolution, respectively.

Electron tunneling is used to explain electronic processes occurring near metal surface. When positively charged ions approach a metal surface, metal valence electrons can neutralize these ions via electron tunneling. For example, in Low-energy ion scattering experiments, low energy ions that are incident on a metal surface are neutralized via electron tunneling [26]. In electrodeposition, metal valence electrons also tunnel to dehydrated metal ions, leading to neutralization $[18,19]$. In electrodeposition the electron tunneling is required for the growth of metal nanowires. This is because the metal nanowires can grow only when the waters of hydration that are attached to the metal ions are displaced on the surface and thus metal atoms incorporate into the ionic metal lattice.

According to the free electron gas model, the metal can be considered a potential box, filled with electrons to the Fermi level, which lies below the vacuum level by several electron volts. The distance from Fermi to vacuum level is called the workfunction, as shown in the left side of figure 4 . In the double layer, there is a very strong electric field

$$
E=V / d
$$

where the $V$ is the applied potential to the metal, assuming that the potential of solution is zero and $d$ the width of the double layer. The potential energy $\varepsilon$ of electrons outside of the metal varies with the field strength $E$ simply as $\varepsilon=$ 
e.E. $x$, as shown in the right side of figure 4. Most of the electron tunneling will occur from the vicinity of the Fermi level where the barrier is thinnest.

It is well-known that increasing the applied potential increases the current density. Here we use the electron tunneling theory to explain this. From eq. (1), we can see that increasing the applied potential will make the electric field strength stronger. This leads to the more rapid change of the potential of electrons outside the metal, as shown in Fig. 4a. In this case, the slope of electron potential with a higher electric field is steeper than that of with a small electric field. The barrier width that electrons tunnel through becomes thinner and electron tunneling occurs more easily. Therefore, the current density increases with increasing the applied potential.
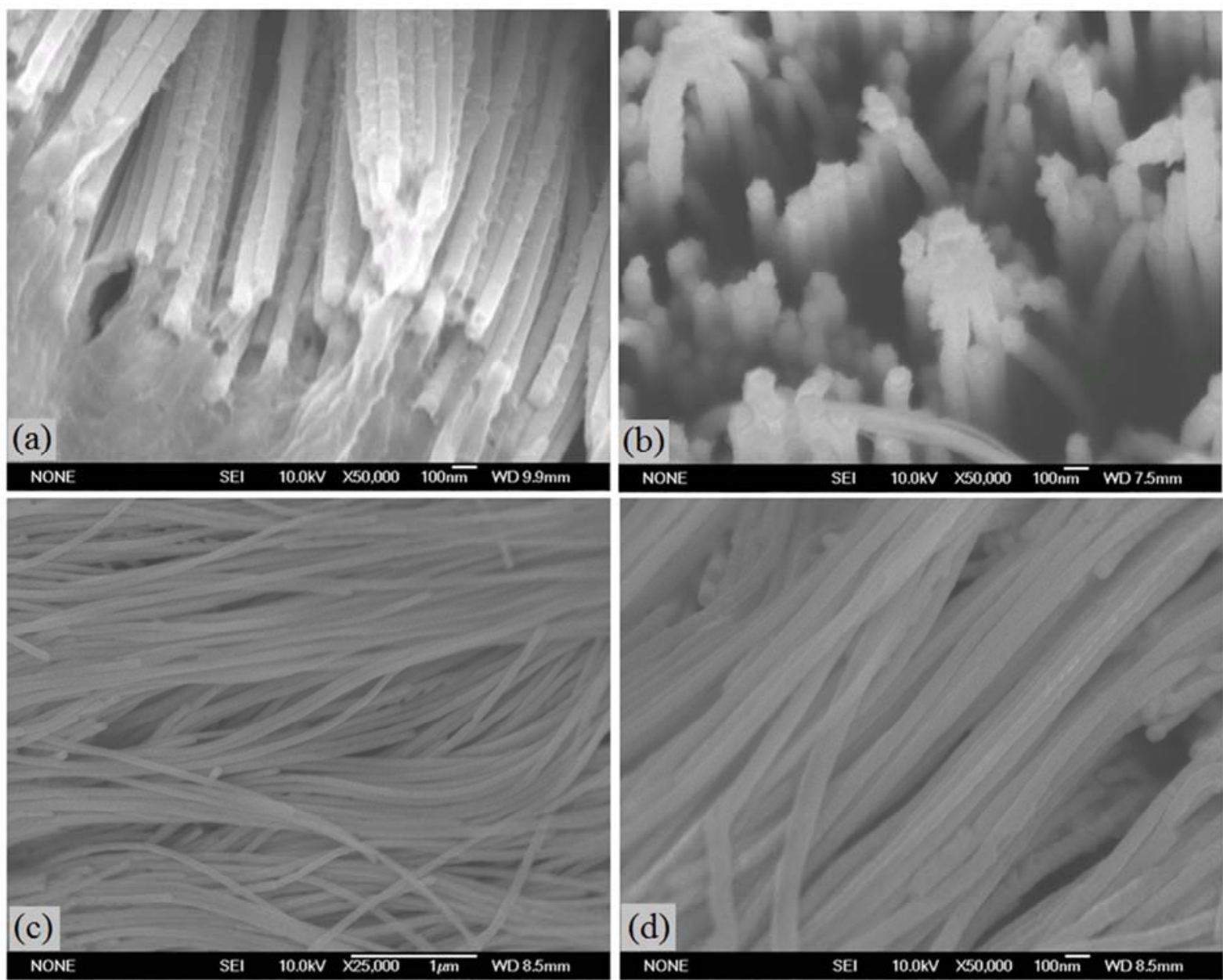

Figure 3. SEM images of Ag nanowires (a, and b), Cu nanowires (c, and d).
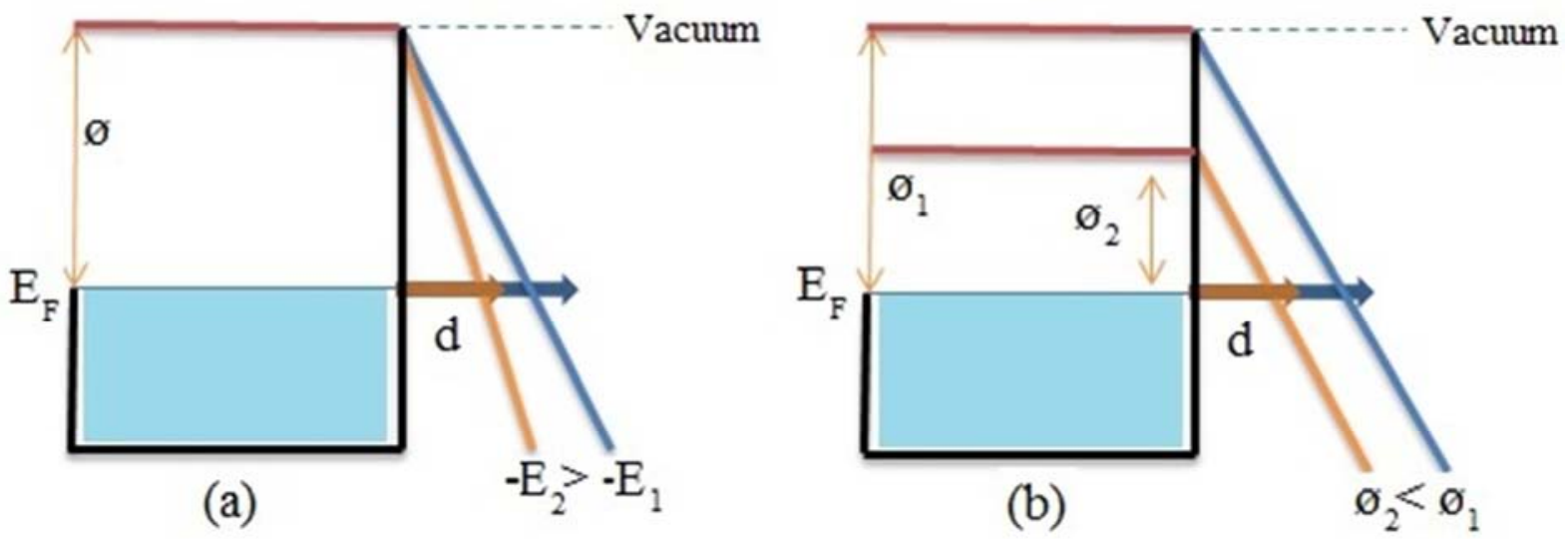

Figure 4. Schematic representation of transmission of electrons near the vicinity of Fermi level $\left(E_{F}\right)$ through the potential barrier: (a) effect of applied field (or electric field strength) on the barrier width (d) of electron tunneling; (b) under the same potential change in barrier width for electron tunneling owing to workfunction. 
The workfunction of metal can have an effect of the barrier width that electrons tunnel through. Fig. $4 \mathrm{~b}$ shows that under the same applied potential (meaning that there is the same electric field strength in the double layer) the barrier width for the metal with small workfunction becomes thinner and electron tunneling takes place more easily. Therefore under the same applied potential, there is a higher current density for deposition of nanowires of the metal with a smaller workfunction [27]. So that is why the deposition current density for $\mathrm{Ag}$ is higher than that of $\mathrm{Cu}$. For the same metal, the planes with a smaller workfunction, such as: (110) plane for both $\mathrm{Ag}$ and $\mathrm{Cu}$ have smaller workfunction's value than of (111) plane, therefore the preferential growth plane is (110) for $\mathrm{Ag}$ and $\mathrm{Cu}$ nanowires.

The physical reason of electron tunneling is that the wavefunction of valence electrons in metals does not drop to zero abruptly at the surface but extend above the surface with an exponentially decaying tail [28]. When the wavefunction tail of the valence electron overlaps with the orbital wavefunction of hydrated metal ions, the valence electron can tunnel to unoccupied orbital of hydrated metal ions. As a first approximation, the tunneling probability is mainly determined by the overlap of the wave functions. The larger the overlap between the orbital wavefunction and the valence electron wavefunction is, the larger the tunneling probability is. The overlap depends very sensitively on the extension of decaying tail and the distance of hydrated metal ions from surface [29, 30].

The wavefunction of electrons above the surface decays exponentially according to [31]

$$
\psi \propto e^{-0.51 \sqrt{\phi} \cdot d}
$$

Where $d$ is the distance from metal surface in $\AA$ and $\varnothing$ the workfunction in $\mathrm{eV}$. From eq. (2) we can see that the valence electron wavefunction of metal with a smaller workfunction decays less steeply and extends more above the surface. The wavefunction overlap depends mainly on the extension of metal wavefunction. As the wavefunction of metal with a smaller workfunction extends more above the surface, the valence electrons of the metal with a smaller workfunction tunnel more easily to hydrated metal ions than those of the metal with a larger function. Thus, we observed a higher current density value when electrodepositing nanowires of the metal with a smaller workfunction.

\section{Conclusions}

Under the same potential and overpotential, the current density of electrodepositing metal nanowires depends on the value of the workfunction of this metal; the smaller the workfunction is, the higher the deposition current density is. The reason for this is that the small workfunction can make the tunneling of electrons easy, thus leading to the faster deposition of metal nanowires. Likewise, for the same metal, plane with smaller work function grow faster than of larger workfunction thus preferential growth plane is (220) for both metals $(\mathrm{Ag}$ and $\mathrm{Cu})$. It is also found that there is no such difference of deposition techniques ( $\mathrm{CV}$ or Fixed potential deposition) on the structure of deposited nanowires.

\section{References}

[1] Chaney SB, Shanmukh S, Dluhy RA, Zhao Y-P. Aligned silver nanorod arrays produce high sensitivity surfaceenhanced Raman spectroscopy substrates. Applied Physics Letters. 2005; 3: 87.

[2] Riveros G, Green S, Cortes A, Gómez H, Marotti RE, Dalchiele EA. Silver nanowire arrays electrochemically grown into nanoporous anodic alumina templates. Nanotechnology. 2006; 17: 561.

[3] Valizadeh S, George JM, Leisner P, Hultman L. Electrochemical synthesis of $\mathrm{Ag} / \mathrm{Co}$ multilayered nanowires in porous polycarbonate membranes. Thin Solid Films. 2002; 402: $262-71$.

[4] Yao JL, Pan GP, Xue KH, Wu DY, Ren B, Sun DM, et al. A complementary study of surface-enhanced Raman scattering and metal nanorod arrays. Pure and Applied Chemistry2000. p. 221.

[5] Cheng Y-H, Cheng S-Y. Nanostructures formed by $\mathrm{Ag}$ nanowires. Nanotechnology. 2004; 15: 171.

[6] Peng Y, Chen Q. Fabrication of one-dimensional $\mathrm{Ag} /$ multiwalled carbon nanotube nano-composite. Nanoscale Research Letters. 2012; 7: 195.

[7] Martin CR. Nanomaterials: A Membrane-Based Synthetic Approach. Science. 1994; 266: 1961-6.

[8] Whitney TM, Searson PC, Jiang JS, Chien CL. Fabrication and Magnetic Properties of Arrays of Metallic Nanowires. Science. 1993; 261: 1316-9.

[9] Wen-Ching Tsai C-CW, and Yung-Yun Wang. Frequency Effect of Pulse Plating on the Uniformity of Copper Deposition in Plated Through Holes. J Electrochem Soc. 2003; 150: 267-72.

[10] A. J. Yin JL, W. Jian, A. J. Bennett, and J. M. Xu. Fabrication of highly ordered metallic nanowire arrays by electrodeposition. Applied Physics Letters. 2001; 79: 1039.

[11] El - Giar EM, Said RA, Bridges GE, Thomson DJ. Localized Electrochemical Deposition of Copper Microstructures. Journal of The Electrochemical Society. 2000; 147: 586-91.

[12] Routkevitch D, Bigioni T, Moskovits $\mathrm{M}, \mathrm{Xu}$ JM. Electrochemical Fabrication of CdS Nanowire Arrays in Porous Anodic Aluminum Oxide Templates. The Journal of Physical Chemistry. 1996; 100: 14037-47.

[13] Ozin GA. Nanochemistry: Synthesis in diminishing dimensions. Advanced Materials. 1992; 4: 612-49.

[14] Tonucci RJ, Justus, B. J., Campillo, A. J., and Ford, C. E. NANOCHANNEL ARRAY GLASS. Science. 1992; 258: 783-5.

[15] Han G C, Zong B Y, H WY. IEEE Trans Magn. 2002: 2562-4. 
[16] D. J. Sellmyer MZ, R. Skomski. Magnetism of Fe, Co, and Ni Nanowires in Self-Assembled Arrays. J Phys Condens Matter. 2001; 13: R433-R60

[17] Tian ML, Wang JG, Kurtz J, Mallouk TE, Chan MHW. Single-Crystal Metal Nanowires via a Two-Dimensional Nucleation and Growth Mechanism. Nano Letters. 2003; 3: 919-23.

[18] Tan M, Chen XQ. Growth Mechanism of Single Crystal Nanowires of fcc Metals ( $\mathrm{Ag}, \mathrm{Cu}, \mathrm{Ni}$ ) and hep Metal (Co) Electrodeposited. Journal of The Electrochemical Society. 2012; 159: K15.

[19] Lower SK. Electrochemistry. Textbook: Simon Fraser University.

[20] Mukhtar A, Shahzad Khan B, Mehmood T. Appropriate deposition parameters for formation of fcc $\mathrm{Co}-\mathrm{Ni}$ alloy nanowires during electrochemical deposition process. Applied Physics A. 2016; 122: 1022.

[21] Mehmood T, Mukhtar A, Wang H, Khan BS. Effect of deposition parameters on the crystal orientation and growth of Ag nanowires. International Journal of Materials Research. 2016; 107: 283-6.

[22] Mehmood T, Shahzad Khan B, Mukhtar A, Chen X, Yi P, Tan M. Mechanism for formation of fcc-cobalt nanowires in electrodeposition at ambient temperature. Materials Letters. 2014; 130: 256-8.

[23] Chelvayohan M, Mee CHB. Work function measurements on
(110), (100) and (111) surfaces of silver. Journal of Physics C: Solid State Physics. 1982; 15: 2305.

[24] Gartland P, Berge S, Slagsvold B. Photoelectric Work Function of a Copper Single Crystal for the (100), (110), (111), and (112) Faces. Physical Review Letters. 1972; 28: 738-9.

[25] Milan Paunovic, Schlesinger M. Fundamentals of Electrochemical Deposition. Fundamentals of Electrochemical Deposition, New York: Wiley. 1998.

[26] Tully JC, N. H. Tolk. In: N. H. Tolk JCT, W. Heiland, C. W. White, editor. Inelastic Particle-Surface Collision. New York: Academic Press INC; 1977. p. 105.

[27] Mehmood T, Mukhtar A, Khan BS, Wu K. Growth Mechanism of Electrodeposited $\mathrm{Fe}$, Co and Ni Nanowires in the Form of Self-Assembled Arrays at Fixed Potential. Int J Electrochem Sci. 2016; 11: 6423-31.

[28] Leemput LECvd, Kempen Hv. Scanning tunnelling microscopy. Rep Prog Phys. 1992; 551165.

[29] Shahzad Khan B, Mehmood T, Mukhtar A, Tan M. Effect of workfunction on the growth of electrodeposited $\mathrm{Cu}, \mathrm{Ni}$ and Co nanowires. Materials Letters. 2014; 137: 13-6.

[30] Khan BS, Mukhtar A, Mehmood T, Tan M. Polarization Curves of Electrodepositing Ag and $\mathrm{Cu}$ Nanowires. Journal of Nanoscience and Nanotechnology. 2016; 16: 9896-900.

[31] Garrett SJ. Introduction to Surface Analysis. the Michigan State University, Chemistry Department, East Lansing. 